\title{
Electroluminescence Efficiency Enhancement in Quantum Dot Light-Emitting Diodes by Embedding a Silver Nanoisland Layer
}

\author{
Xuyong Yang, Pedro Ludwig Hernandez-Martinez, Cuong Dang, Evren Mutlugun, \\ Kang Zhang, Hilmi Volkan Demir,* and Xiao Wei Sun**
}

A colloidal quantum dot light-emitting diode (QLED) is reported with substantially enhanced electroluminescence by embedding a thin layer of $\mathrm{Ag}$ nanoislands into hole transport layer. The maximum external quantum efficiency (EQE) of $7.1 \%$ achieved in the present work is the highest efficiency value reported for green-emitting QLEDs with a similar structure, which corresponds to $46 \%$ enhancement compared with the reference device. The relevant mechanisms enabling the EQE enhancement are associated with the near-field enhancement via an effective coupling between excitons of the quantum dot emitters and localized surface plasmons around Ag nanoislands, which are found to lead to good agreement between the simulation results and the experimental data, providing us with a useful insight important for plasmonic QLEDs. solution-processed preparation techniques, ${ }^{[8-10]}$ which make QDs one of the most promising materials for use in light-emitting diodes (LEDs). Since the first report of electrically driven quantum dot LEDs (QLEDs) utilizing CdSe QDs in $1994,{ }^{[11]}$ QLEDs have received considerable attention for academic research and potential commercialization. After about two decades of research and development, the full-color and saturated electroluminescence (EL) spectra from QLEDs have been achieved. Currently, QLEDs are reaching the performance of organic LEDs (OLEDs) and emerging as a candidate for single-material, full-color light sources. ${ }^{[12-17]}$ However, although rapid progress has been made in the device performance owing to the technological development and accumulation of relevant knowledge in materials and device architectures, further improvements in device efficiency are still necessary for the widespread use and commercialization of QLED technology.

The coupling between excitons and surface plasmons (SPs) by the incorporation of metallic nanostructures into light-emitting devices is becoming one of the most promising approaches for the enhancement of internal quantum efficiency in LEDs. ${ }^{[18-24]}$ SPs are collective oscillations of free electrons at the interface between a metal and a dielectric material. The plasmonic coupling effect between excitons and SPs caused by the overlap of the local electromagnetic field of excitons in the emissive layer and SPs can lead to significant enhancement in radiative recombination rate through effective energy transfer in LEDs. Recently Choi et al. ${ }^{[18]}$ have reported highly efficient ploymer LEDs (PLEDs) through local surface plasmon resonance (LSPR) enhancement with carbon-dot supported silver nanoparticles (CD-Ag NPs) and Xiao et al.[19] demonstrated the surface plasmon-enhanced EL in OLEDs by incorporating $\mathrm{Au}$ NPs in the hole injection layer of polyethylene dioxythiophene:polystyrene sulfonate (PEDOT:PSS). On the other hand, the large QD photoluminesence (PL) enhancements through QD-metal nanostructure interactions have been intensively demonstrated in the past few years, ${ }^{[25-29]}$ suggesting the great potential that properly tailored plasmonic nanostructures in QLEDs could be used to enhance device efficiency.

DOI: 10.1002/adom.201500172 
Here, we report the first account of plasmon-enhanced EL in QLEDs by embedding Ag nanoislands into an organic hole transport layer. The resulting plasmonic QLEDs exhibit superior performance compared to that of the reference QLEDs without Ag nanoislands. The metallic Ag is used in the present study due to the sharpest and strongest bands among all noble metals. To our knowledge, the external quantum efficiency (EQE) of 7.1\% achieved in this work corresponds to the highest reported EQE value for green-emitting QLEDs with an inverted device structure (without outcoupling structures). The relevant mechanisms enabling the enhanced efficiency in QLEDs are discussed in detail.

\section{Results and Discussion}

Figure 1a shows the atomic force microscopy (AFM) image of the Ag nanoisland film thermally evaporated on ITO with an average size of $20 \mathrm{~nm}$ (this morphology of $\mathrm{Ag}$ nanoislands is formed when Ag film thickness is below $10 \mathrm{~nm}$. ${ }^{[30]}$ The influence of silver particle size on surface plasmon, the important parameter for the study of LSPR, is illustrated in Figure 1b. The absorption peak redshifts from 460 to $580 \mathrm{~nm}$ with increasing particle size of Ag nanoislands. The plasmonic QLEDs with the green $\mathrm{QD}$ emission have been fabricated to investigate the capability of $\mathrm{Ag}$ nanoislands to enhance the device performance. The QLED architecture used is ITO/ZnO NPs
$(40 \mathrm{~nm}) / \mathrm{QDs}(20 \mathrm{~nm}) / 2,2^{\prime}, 2^{\prime \prime}$-tris-( $N$-carbazolyl)-triphenylamine $(\mathrm{TCTA}, \quad \times \quad \mathrm{nm}) / \mathrm{Ag}$ nanoislands/TCTA $(60-\times \mathrm{nm}) / \mathrm{MoO}_{3}$ $(10 \mathrm{~nm}) / \mathrm{Al}(100 \mathrm{~nm})$, as shown in Figure 1c. Other than the $\mathrm{MoO}_{3}$, Ag nanoislands and $\mathrm{Al}$ anode, which were deposited by vacuum thermal evaporation, all other layers were sequentially deposited onto ITO using spin-coating. The TCTA film used in the device not only acts as a hole transport layer but also as a dielectric spacer for adjusting the distance between the QDs and Ag nanoislands for avoiding the fluorescence quenching due to their extraordinarily high molar extinction coefficients and broad energy bandwidth of metals. ${ }^{[31]}$ The enhancement of EL in QLEDs will be maximized when they are excited at the frequency of surface plasmon resonance in Ag nanoisland film. The PL emission peak of the QDs in toluene used here is at $\approx 502 \mathrm{~nm}$, which can match the absorption peak of Ag nanoislands by tuning the particle size of Ag nanoislands (Figure 1d). Therefore, a maximum coupling between the EL of QLED device and the SPs of Ag nanoisland layer can be expected.

Figure 2a presents the EL spectra from the QLED incorporating a silver nanoisland layer (the thicknesses of the QD and ZnO NP layers are 20 and $40 \mathrm{~nm}$, respectively) and the reference device without $\mathrm{Ag}$ nanoisland layer. The pure green QD emissions at a peak wavelength of $» 508 \mathrm{~nm}$ are achieved in both of the devices, which illustrates the highly efficient recombination of electrons and holes in the QD emissive layer. It can be noted that the EL emission wavelength is red-shifted $(\gg 6 \mathrm{~nm})$ from the PL emission of QD solution resulting from a
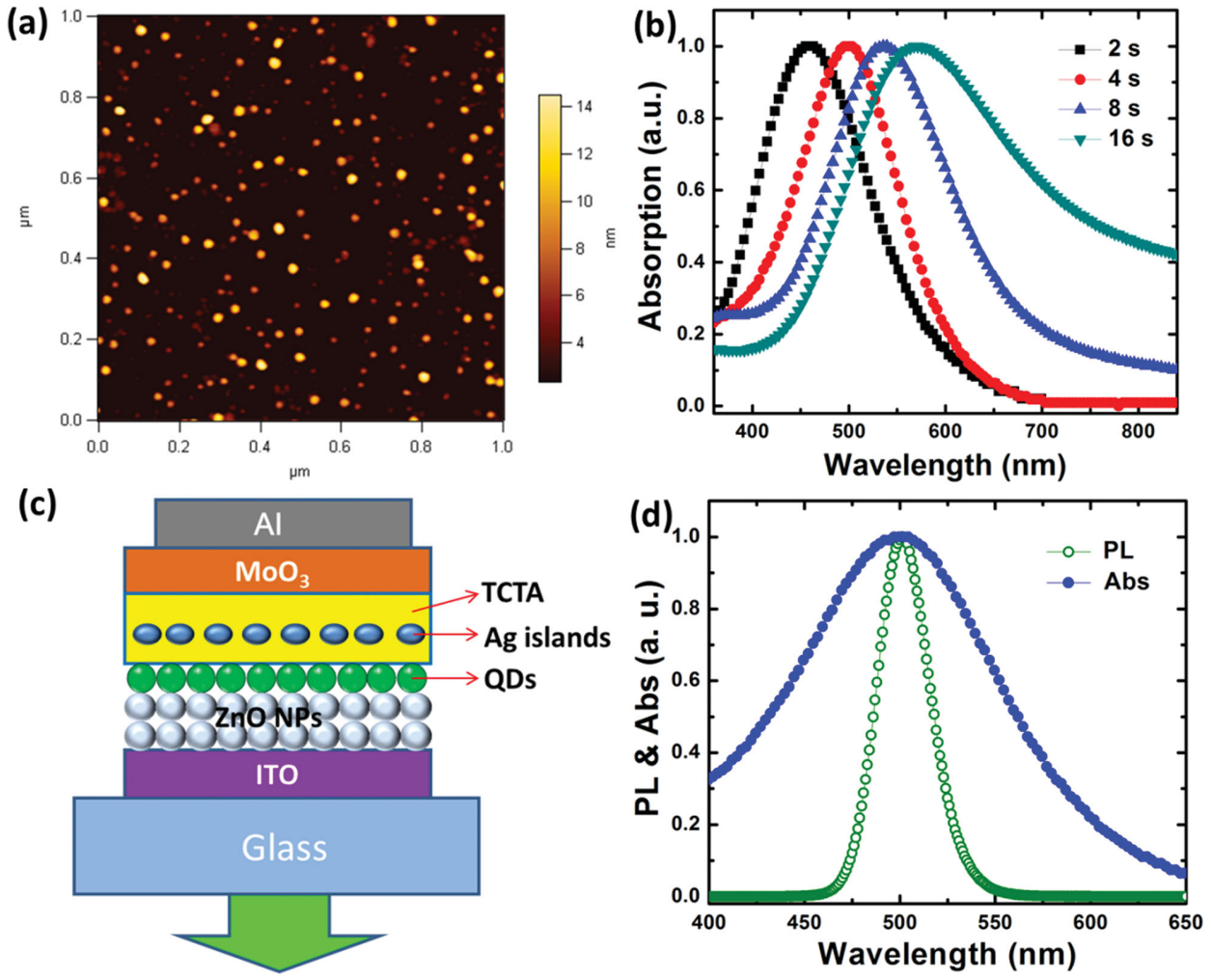

Figure 1. a) AFM image of Ag nanoislands formed on ITO substrate. b) Absorption spectra of Ag nanoislands with different deposition time. The Ag nanoislands were deposited with a fast deposition rate of $0.375 \mathrm{~nm} \mathrm{~s}^{-1}$. c) Schematic of the device configuration of the QLEDs embedded Ag nanoislands. d) Absorption spectrum of the Ag nanoislands and PL spectrum of the green QDs excited at $400 \mathrm{~nm}$. 

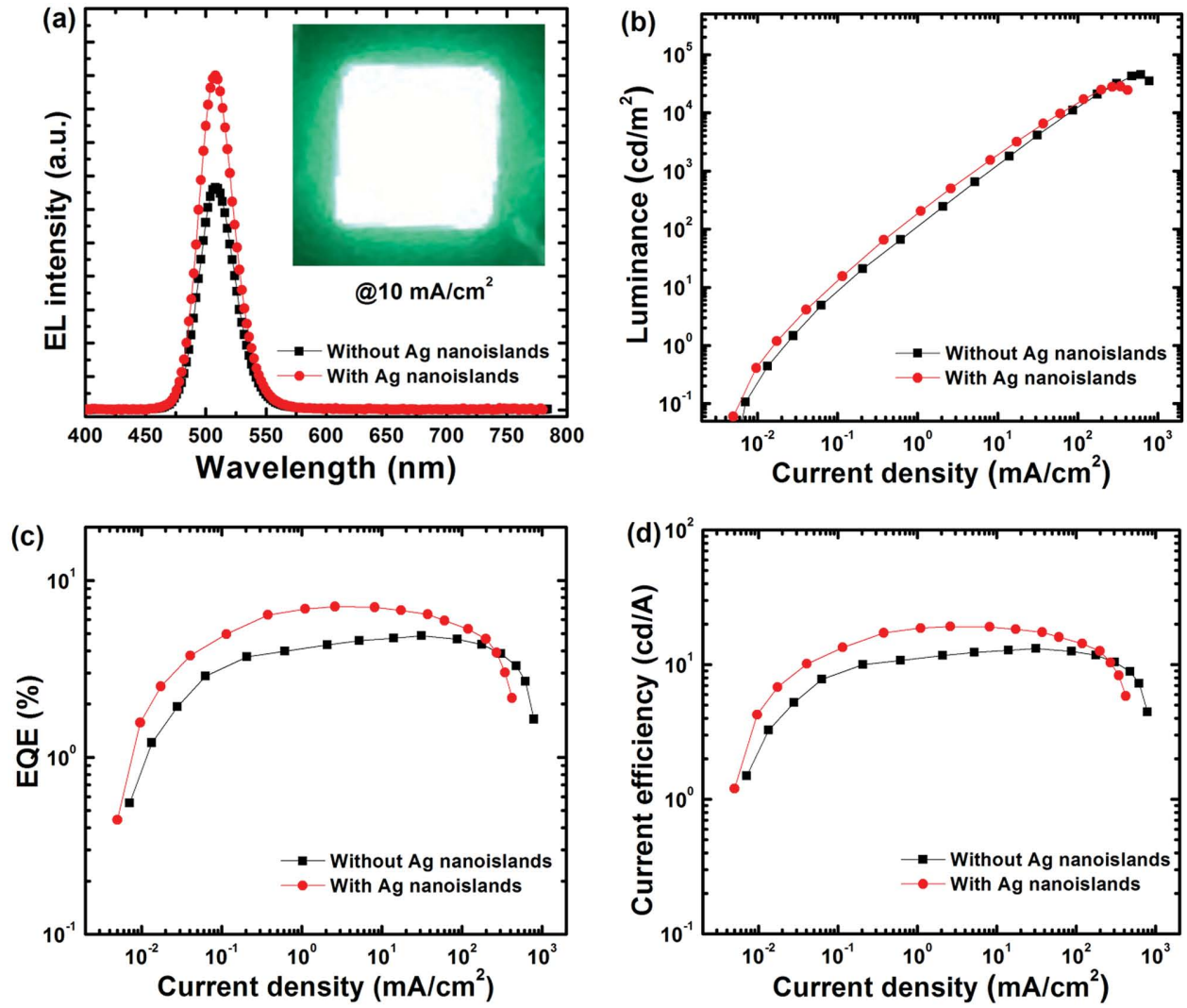

Figure 2. a) EL spectra of a typical plasmonic QLED with $\mathrm{Ag}$ nanoislands and the reference device without $\mathrm{Ag}$ nanoislands. Inset: The photographic image of the plasmonic QLED. Comparison of b) luminance, c) EQE, and d) CE for the plasmonic and reference devices as a function of the current density.

combination of finite dot-to-dot interaction in close packed solid films and the electric-field-induced Stark effect. However, a significant EL emission enhancement without obvious spectral change is observed in the resulting QLED with the incorporation of Ag nanoislands. This is because the resonance energy of the SP excitation mode matches with the excitons of QDs in the plasmonic QLED, leading to an effective SP enhancement by Ag NPs. A corresponding image of the plasmonic QLED output was recorded at the luminance of $200 \mathrm{~cd} \mathrm{~m}^{-2}$, which displays a uniform, bright and saturated green emission profile (inset of Figure 2a). The EL characteristics for the QLEDs with and without Ag nanoislands are plotted in Figure $2 \mathrm{~b}-\mathrm{d}$. The typical current density and luminance curves as a function of the applied voltage for the two devices are depicted in Figure $2 \mathrm{~b}$. The QLED with Ag nanoislands exhibits a higher brightness before the plasmonic device reaches its maximum luminance of $28500 \mathrm{~cd} \mathrm{~m} \mathrm{~m}^{-2}$ compared with the reference device. The external quantum efficiency-current density (EQE-J) and current efficiency-current density (CE-J) characteristics are shown in Figure 2c,d. With the incorporation of $\mathrm{Ag}$ nanoislands in TCTA, the device efficiency for the plasmonic QLED is dramatically enhanced, with a maximum EQE of $7.11 \%$ and CE of $19.2 \mathrm{~cd} \mathrm{~A}^{-1}$, respectively, significantly higher than that of the reference device $\left(4.86 \%\right.$ and $\left.13.2 \mathrm{~cd} \mathrm{~A}^{-}\right)$, which are the highest reported efficiency values for green QLEDs with an inverted structure (without outcoupling medium). ${ }^{[32,33]}$
To investigate the LSPR effect of the Ag nanoislands on the fluorescence from QD layer in QLEDs, fluorescence-lifetime imaging microscopy (FLIM) and time-resolved fluorescence spectroscopy are carried out on the CdSe/ZnS QD films covered with Ag nanoislands (the thickness of TCTA between QDs and $\mathrm{Ag}$ nanoislands is $25 \mathrm{~nm}$ ). The FLIM image of the sample (one half is deposited with Ag nanoislands and the other half is without Ag nanoislands). Figure 3a clearly shows the distinctive regions of lifetimes with and without $\mathrm{Ag}$ nanoislands. It can be seen that the region with Ag nanoislands is much darker due to the excited state lifetime of the QDs decreasing with the increase in coupling between the exciton and surface plasmon. Accordingly, it can be seen from Figure $3 \mathrm{~b}$ that there are two main lifetime distribution regions in the sample, which correspond to the region with $\mathrm{Ag}$ nanoislands (peaking at $\gg 8.3 \mathrm{~ns}$ ) and without Ag nanoislands (peaking at »11.2 ns), respectively. The arbitrarily selected two PL lifetimes from the two different regions are shown in Figure 3c. The lifetime of the QD excitons $\left(\tau_{\text {exciton }}\right)$ in the presence of Ag nanoislands is significantly shortened from 11.08 to $7.31 \mathrm{~ns}$. Meanwhile, as expected from the PL spectra of the QD films with and without Ag nanoislands measured at room temperature (Figure 3d), the PL intensity for the QD film with Ag nanoislands is obviously enhanced compared to that of the QD film without Ag nanoislands, which indicates that the excitons of QDs are effectively excited at the frequency of surface plasmon resonance in Ag nanoisland films. 

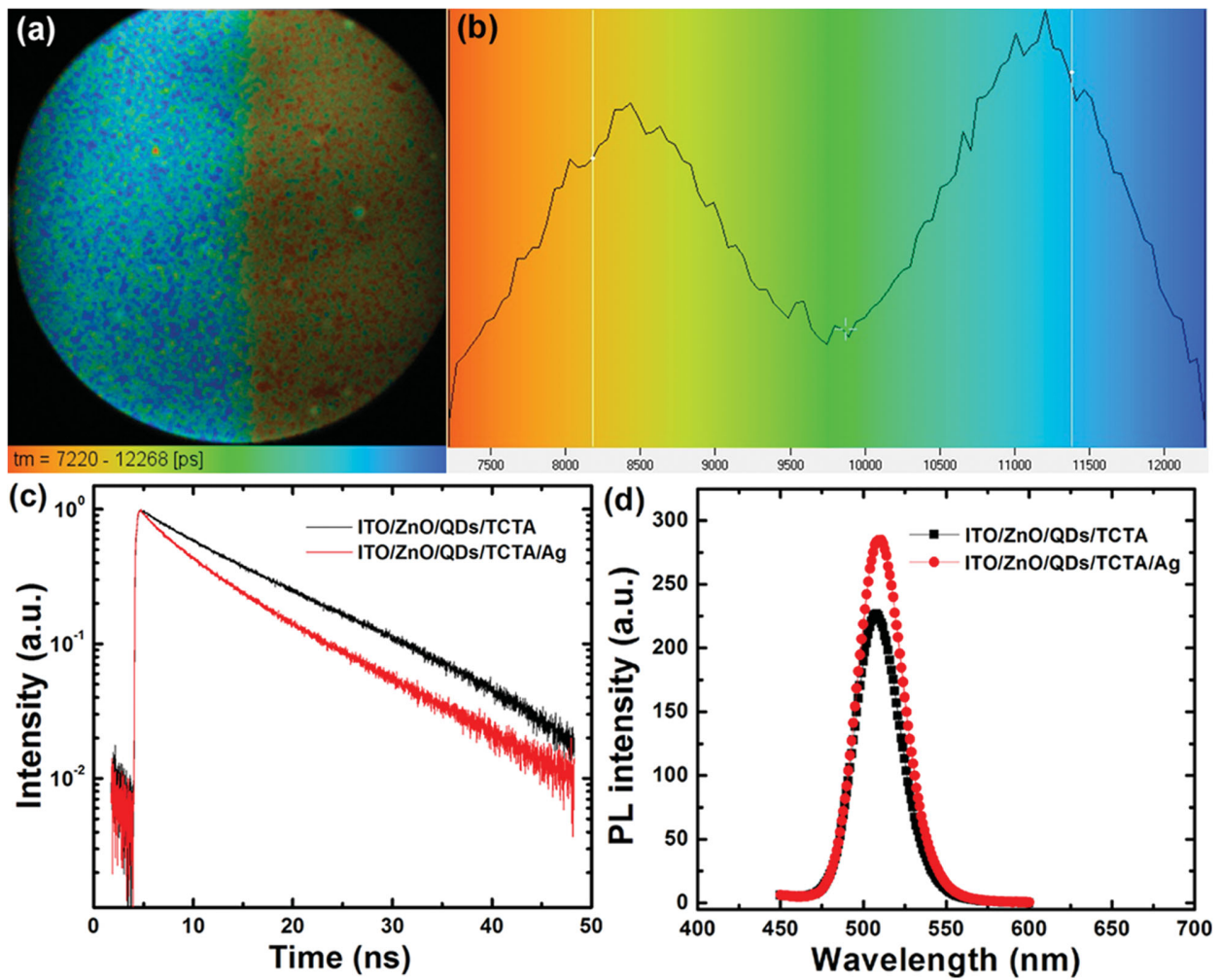

Figure 3. a) FLIM image of CdSe/ZnS QDs films recorded upon excitation at $400 \mathrm{~nm}$. The QDs layer without Ag nanoislands is shown in the bright color while QDs covered by Ag nanoislands is shown in the dark color. b) Statistical lifetime distribution (ns) within the whole area of measurement. The lifetime of QDs covered by Ag nanoislands is shortened. c) Time-resolved fluorescence spectra of QD films with and without nanoislands. d) PL intensity of QD films with and without Ag nanoislands excited under $400 \mathrm{~nm}$.

To theoretically estimate the EL emission enhancement factor, we calculate the electric field variation of QD layer in the presence of Ag nanoislands due to the plasmonic effects. The morphology of the Ag nanoislands in this work is assumed to be spherical (the ratio of diameter to height for Ag nanoislands is less than 2). The main interaction in the optical properties of a complex structure composed of metal nanoparticles (MNPs) and semiconductor QDs is exciton-plasmon coupling. The following subsection describes this particular case.

Here we consider a single QD interacting with a silver nanoisland in the presence of a constant electric field, as shown in Figure 4. Within the simplest rate model, the number of excitons $\left(N_{\text {exc }}\right)$ trapped in the QD, under constant exciton generation rate, is given by

$$
-\left(\gamma_{\mathrm{r}}+\gamma_{\mathrm{nr}}+\gamma_{\mathrm{nr}, \mathrm{metal}}\right) N_{\mathrm{exc}}+I_{\mathrm{Gen}}=0
$$

where $I_{\mathrm{Gen}}$ is the exciton generation rate in the $\mathrm{QD}, \gamma_{\mathrm{r}}(\mathrm{nr})$ is the QD radiative (nonradiative) rate, and $\gamma_{n r \text {,metal }}$ is the exciton recombination rate of the QD because of energy transfer to the MNP. $\gamma_{\mathrm{QD} \rightarrow \mathrm{MNP}}^{\text {trans }}$ is calculated by ${ }^{[34]}$

$\gamma_{\mathrm{QD} \rightarrow \mathrm{MNP}, \alpha}^{\mathrm{trans}}=\frac{2}{\hbar} b_{\alpha}\left(\frac{e d_{\mathrm{exc}}}{\varepsilon_{\text {eff }}}\right)^{2} \frac{R_{\mathrm{MNP}}^{3}}{d^{6}}\left|\frac{3 \varepsilon_{0}}{2 \varepsilon_{0}+\varepsilon_{\mathrm{MNP}}(\omega)}\right|^{2} \operatorname{Im}\left|\varepsilon_{\mathrm{MNP}}(\omega)\right|$

where $b_{\alpha}=\frac{1}{3} \cdot \frac{1}{3}, \frac{4}{3}$ for $\alpha=x, y, z$, respectively, $\varepsilon_{0}$ is the dielectric constant of the outside medium, $\varepsilon_{\mathrm{MNP}}(\omega)$ is the dielectric function of the MNP, $R_{\mathrm{MNP}}$ is the MNP radius, $d_{\mathrm{exc}}$ is the exciton dipole moment in the QD, $d$ is the center-to-center separation distance between the QD and MNP, and $\varepsilon_{\text {eff }}$ is the effective dielectric constant given by a)

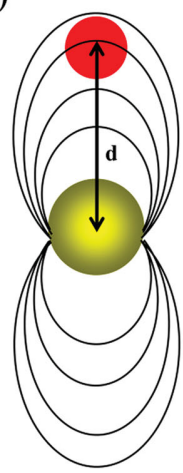

b)

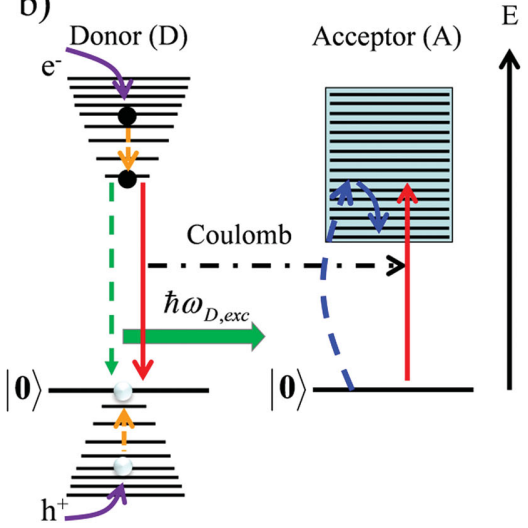

Figure 4. a) Surface plasmon enhanced QD emission. b) Energy diagram for the donor-acceptor (D-A) pair energy transfer process. Purple solid line represents the electron and hole injection. Orange dashed line represents the electron and hole relaxation process. Green dashed lines show light emission process (relaxation from the lowest excited state to the ground state). Red solid lines represent the energy transfer from the donor to the acceptor. Horizontal dashed black line shows the Coulomb interaction between the donor and acceptor. Blue dashed lines represent the absorption process of the acceptor. 
$\varepsilon_{\text {eff }}=\frac{2 \varepsilon_{0}+\varepsilon_{\mathrm{QD}}}{3}$

We assume that the intrinsic nonradiative rate is not affected by the $\operatorname{MNP}\left(\gamma_{\mathrm{nr}}=\gamma_{0, \mathrm{nr}}\right)$, whereas the intrinsic radiative rate is modified by the MNP. This assumption is valid because the experiment is set up to enhance the emission of the QD in the presence of MNP. The radiative rate is modified by ${ }^{[35]}$

$\gamma_{\mathrm{r}}=A\left(\omega_{\mathrm{emiss}}\right) \gamma_{0, \mathrm{r}}$

where $\gamma_{0, \mathrm{r}}, \gamma_{0, \mathrm{nr}}$ are the parameters in the absence of MNP; $\omega_{\text {emiss }}$ is the exciton-emission frequency; and $A(\omega)$ is the electric field enhancement factor defined as ${ }^{[36]}$

$A(\omega)=\frac{\left|E_{\text {in,QD }}\right|^{2}}{\left|E_{0}\right|^{2}}$

where $E_{\mathrm{in}, \mathrm{QD}}$ is the electric field inside the QD due to the presence of MNP and $E_{0}$ is the electric field inside the QD in the absence of MNP. For the electric field enhancement factor calculation, the QD is assumed to be a point-like dipole. The EL enhancement factor is defined as

$\eta_{\mathrm{EL}}=\frac{Y_{\mathrm{MNP}}\left(\omega_{\mathrm{emiss}}, \mathrm{r}\right)}{Y_{0}}$

where $Y_{\mathrm{MNP}}\left(Y_{0}\right)$ is the quantum yield of QD film in the presence (absence) of MNP, respectively; and $\gamma_{0, \mathrm{r}}=Y_{0} \gamma_{0, \mathrm{exc}}, \gamma_{0, \mathrm{nr}}=\left(1-Y_{0}\right) \gamma_{0, \mathrm{exc}} \cdot \gamma_{0, \mathrm{exc}}$ is the exciton recombination rate in the absence of MNP. After simply algebra, the EL enhancement boils down to

$$
\eta_{\mathrm{EL}}=\frac{A\left(\omega_{\text {emiss }}, \mathrm{r}\right)}{\left[A\left(\omega_{\text {emiss }}, \mathrm{r}\right) Y_{\mathrm{QD}}+\left(1-Y_{\mathrm{QD}}\right)+\frac{\gamma_{\mathrm{QD} \rightarrow \mathrm{MNP}}^{\text {trans }}\left(\omega_{\text {emiss }}, \mathrm{r}\right)}{\gamma_{0, \text { tot }}}\right]}
$$

is the final expression for the enhancement factor.

Since the strong distance dependent coupling between MNPs and excitons, the thickness of TCTA as a spacer between the QDs and Ag nanoislands has been adjusted to achieve the best device performance. Figure $\mathbf{5}$ shows the enhanced EL emission resulting from the nonradiative energy transfer (NRET) for QDs to Ag nanoislands when the donor (QDs) is coupled to the acceptor (Ag nanoislands). As shown in Figure 5a, we observe a maximum emission enhancement factor of 1.46 when the surface-to-surface distance between the QD and Ag nanoisland is $25 \mathrm{~nm}$. This result is comparable to the simulation value of 1.7 when the center-to-center distance between the QD and $\mathrm{Ag}$ nanoisland is $18 \mathrm{~nm}$ (Figure $5 \mathrm{~b}$ ). The parameters used in the simulation calculation are: $R_{\mathrm{MNP}}=10 \mathrm{~nm}, R_{\mathrm{QD}}=3.5 \mathrm{~nm}$, $\lambda_{\text {emiss }}=508 \mathrm{~nm}, Y_{0}=0.25, \tau_{0, \text { exc }}=11.08 \mathrm{~ns}$, and $\varepsilon_{\mathrm{MoO}_{3}}=3.0$.

The improved QD EL emission could be explained as follows: the localized surface plasmon in the MNPs enhances the QD radiative channel, allowing the QDs to emit more photons per unit time. In other words, more electron-hole pairs can be injected to the QDs in the same period of time as compared to the case without MNPs. However, the overall quantum efficiency of QDs decreases in the presence of MNPs because of the energy transfer between the QDs and MNPs. Thus, there are competing effects between the enhanced radiative channel and the energy transfer between QD and MNPs. It can be noted that in our calculation we predicted a center-to-center distance of $18 \mathrm{~nm}$ with a maximum enhancement factor of 1.7 whereas in the experiment we measured a surface-to-surface distance of $25 \mathrm{~nm}$ with an enhancement factor of 1.46. This discrepancy between the theory and experimental value is mainly due to the fact that in the calculation we considered the interaction between a single QD and a single MNP; and we approximated the Ag nanoisland to a spherical-like MNP. It may not be the case in the experiment and multiple interactions between QDs and Ag nanoislands need to be considered. Despite the differences, our calculation gives the trend for the experimental results, which we believe pinpoint some of the main features involved in the experiment. In addition, it is worth mentioning that the actual thickness of the TCTA as a spacer should be shorter than the proposed value $(25 \mathrm{~nm})$ due to the diffusion of Ag nanoislands into TCTA under a high deposition temperature. Since the diffusion depth is also strongly dependent on the properties of organic transport layers used, ${ }^{[37]}$ the different organic HTLs (TCTA, CBP, and NPB) are studied in our work and it is found that TCTA plays a better role in improving device performance (Figure S1, Supporting Information). This
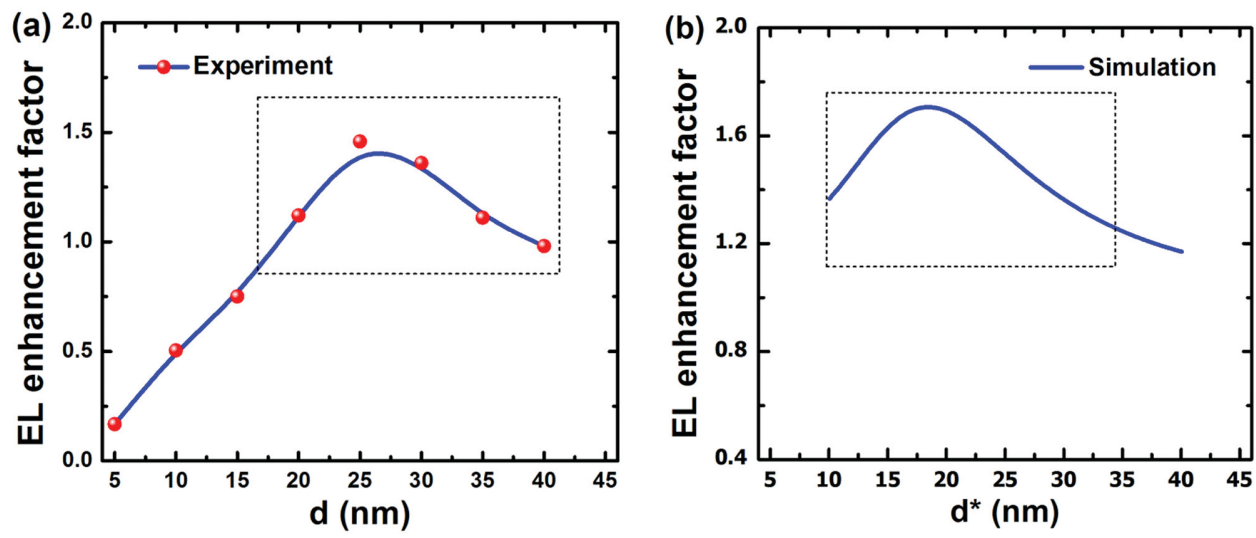

Figure 5. a) Experiment and b) simulation results. Electroluminescence enhancement factor for the QDs in the presence of Ag nanoislands. $d$ and $d *$ present the surface-to-surface and center-to-center distance between the QDs and MNPs, respectively. 
can be attributed to the shorter diffusion depth for Ag nanoislands into TCTA due to the relatively high melting point of TCTA. Accordingly, the radiative decay rate for the optimized QLED is changed from $0.0226 \mathrm{~ns}^{-1}$ (without MNP) to $0.106 \mathrm{~ns}^{-1}$ (with MNP) according to our calculation (Figure S2, Supporting Information). The radiative decay rate with MNP is more than four time faster than the radiative decay rate without MNP, which can be attributed to an increase in the QD's density of states due to the strong electric field coming from the localized surface plasmon.

Figure 6 shows the current density-voltage $(J-V)$ characteristics for these two devices with and without Ag nanoislands. Electrical characterization results revealed that the presence of the Ag nanoislands in device has a little effect on the charge transport process that the current injection for the device with $\mathrm{Ag}$ nanoislands is slightly less efficient compared to that of the reference device. In addition, it is found that the current injection capacity will be sharply decreased with increasing the thickness of Ag film (Figure S3, Supporting Information). The reason behind the decrease in current density for the QLEDs with the incorporation of $\mathrm{Ag}$ nanoislands may be caused by the trapping of holes in Ag nanoislands. ${ }^{[38]}$ As shown in the schematic energy level diagram of the device (inset of Figure 6), after the incorporation of Ag nanoislands in QLEDs, although most of holes injected via carrier breakthrough from the $\mathrm{MoO}_{3}$ /TCTA junction can go through the thin and discontinuous Ag nanoisland layer, minority holes are still trapped in Ag nanoislands with a high work function, resulting in the reduced hole injection into QD layer. Therefore, in order to reduce the negative impact of $\mathrm{Ag}$ nanoislands in the diode on electrical properties of device to the maximum extent for obtaining the best device performance, the Ag nanoisland layer with a deposition time of $2 \mathrm{~s}$ is used in our present study even if the spectrum overlap between the PL of the green QDs

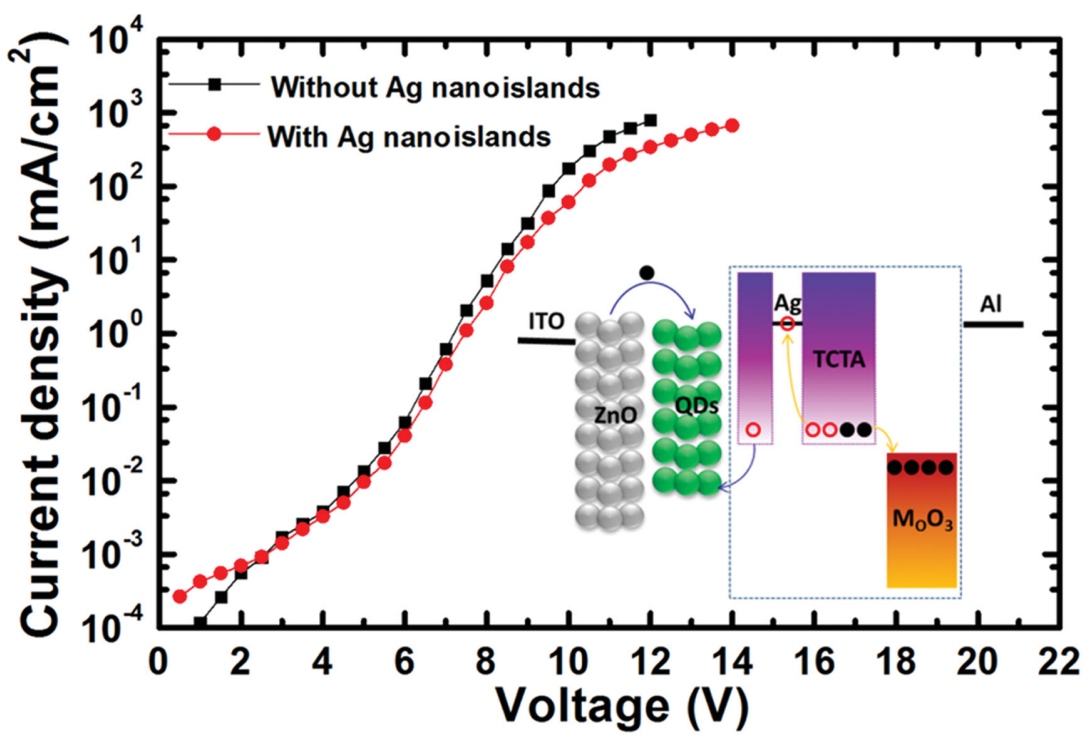

Figure 6. Electric characteristics of QLEDs with and without Ag nanoislands. Inset: Energy level diagram of the QLEDs embedded Ag nanoislands. Red hollow circles represent holes. The hole injection from anode to the TCTA results from carrier breakthrough from the $\mathrm{MoO}_{3} / \mathrm{TCTA}$ junction. Black solid circles represent electrons. The electrons are injected from the Al to the QD layer via the conduction band of the $\mathrm{ZnO}$ nanoparticle layer. and the absorption for Ag nanoisland layer with a deposition time of $4 \mathrm{~s}$ is larger.

\section{Conclusions}

In summary, we have demonstrated the enhanced electroluminescence in QLEDs with an emission enhancement factor of 1.46 by incorprating plasmonic Ag nanoislands into device structure. The maximum EQE of 7.1\% for the resulting QLEDs with Ag nanoislands exceeds that of the best QLEDs based on the same emitting layer and optimized transport layers. The significant light emission enhancement is mainly attributed to the enhanced radiative decay rate in the QD layer as a result of the LSPR-induced near-field enhancement in the QD layer because of the surrounding Ag nanoislands. Measurements of reduced fluorescence lifetime and enhanced emission intensity in the presence of $\mathrm{Ag}$ nanoislands further support the strong exciton-plasmon coupling. These results suggest that the noble metal nanostructures have great potential in the application of QLEDs.

\section{Experimental Section}

Preparation of CdSe/ZnS QDs and ZnO Nanoparticles: Green-emitting $\mathrm{CdSe} / \mathrm{ZnS}$ QDs were synthesized according to a method reported in the literature. ${ }^{[39]}$ For a typical QD preparation, $0.1 \mathrm{mmol}$ of cadmium oxide (CdO), $4 \mathrm{mmol}$ of zinc acetate $\left(\mathrm{Zn}(\text { Acet })_{2}\right)$, and $5 \mathrm{~mL}$ of oleic acid (OA) were loaded in a $50 \mathrm{~mL} 3$-neck flask, heated to $150{ }^{\circ} \mathrm{C}$ under vacuum to form cadmium oleate $\left(\mathrm{Cd}(\mathrm{OA})_{2}\right)$ and zinc oleate $\left(\mathrm{Zn}(\mathrm{OA})_{2}\right)$. Next, $20 \mathrm{~mL}$ of 1-octadecene (1-ODE) was added to the reaction flask, and the reactor was filled with nitrogen. After that, the reaction mixture was heated up to $300{ }^{\circ} \mathrm{C}$. At the elevated temperature, $1.6 \mathrm{~mL}$ of tri-n-octylphosphine (TOP) with $0.15 \mathrm{mmol}$ of selenium (Se) and $4 \mathrm{mmol}$ of sulphur (S) dissolved was injected into the flask swiftly. The reaction mixture was kept at $300{ }^{\circ} \mathrm{C}$ for 10 min for the QD growth. To purify the as-prepared QDs, the reaction mixture was cooled down to room temperature, and the QDs were extracted by the addition of acetone and methanol, followed by centrifugation. The purified QDs with an average particle size of $\gg 7 \mathrm{~nm}$ was measured to give a quantum yield of $\gg 60 \%$ in toluene by comparing the fluorescence intensities with a standard reference dye, fluorescein-27. ZnO nanoparticles (NPs) were synthesized with a mean diameter of 3-4 $\mathrm{nm}$ based on a previously reported solution-precipitation method. ${ }^{[40]}$

Fabrication of Plasmonic QLED Devices: The patterned ITO substrates were cleaned by sonication sequentially in detergent, deionized water, acetone, and isopropyl alcohol. The ZnO NP layer was then spin-coated on the ITO substrate from $\mathrm{ZnO}$ ethanol solution at $1000 \mathrm{rpm}$ for $60 \mathrm{~s}$ and annealed at $150^{\circ} \mathrm{C}$ for $30 \mathrm{~min}$ in a nitrogen glove box. Next, the QD layer was deposited on $\mathrm{ZnO} \mathrm{NP}$ layer by spin-coating the QD dispersion ( $15 \mathrm{mg} \mathrm{mL}^{-1}$ in toluene) at a rate of $2000 \mathrm{rpm}$ for $60 \mathrm{~s}$, and cured at $90^{\circ} \mathrm{C}$ for $30 \mathrm{~min}$. Subsequently, the $4,4^{\prime}-N, N^{\prime}$-dicarbazole-biphenyl (TCTA, $25 \mathrm{~nm}$ ) is were first thermally deposited on QD layer under a base pressure of $\gg 2 \times 10-4 \mathrm{~Pa}$, and then the $\mathrm{Ag}$ nanoislands were quickly deposited. 
After that, the TCTA $(35 \mathrm{~nm}), \mathrm{MoO}_{3}(10 \mathrm{~nm})$, and $\mathrm{Al}(190 \mathrm{~nm})$ layers were deposited in sequence.

Instrumentation: The EL spectra of QLEDs were measured using a Photo Research PR705 Spectra Scan spectrometer and the measurement for overall device performances were made using a technique described by Forrest et al. in which a photodiode was placed in contact with the active pixel (for which the area of the photodiode is much larger than that of the active pixel). The EL emission from QLEDs was first measured as the photodiode current and the current of device was also obtained simultaneously. The total device lumiance and efficiency were calculated by these two quantities together with the integral EL spectra. The PL spectra were recorded using a Shimadzu RF-5301PC spectrofluorophotometer equipped with a $150 \mathrm{~W}$ Xenon lamp as the excitation source. Atomic force microscopy (Cypher AFM, Asylum Research) was used to obtain the surface morphology of the $\mathrm{Ag}$ nanoisland layers on ITO substrates. All measurements were carried out at room temperature under ambient conditions.

\section{Supporting Information}

Supporting Information is available from the Wiley Online Library or from the author.

\section{Acknowledgements}

The authors would like to thank the financial support from Singapore National Research Foundation under NRF-RF-2009-09, NRF-CRP-6-2010-02, and NRF-CRP-11-2012-01 and the Science and Engineering Research Council, Agency for Science, Technology and Research (A*STAR) of Singapore (Project Nos. 0921010057 and 112120 2009). H.V.D also acknowledges ESF EURYI and TUBA.

Received: March 29, 2015

Revised: April 22, 2015

Published online: May 13, 2015

[1] O. Chen, J. Zhao, V. P. Chauhan, J. Cui, C. Wong, D. K. Harris, H. Wei, H.-S. Han, D. Fukumura, R. K. Jain, M. G. Bawendi, Nat. Mater. 2013, 12, 445

[2] J. M. Pietryga, D. J. Werder, D. J. Williams, J. L. Casson, R. D. Schaller, V. I. Klimov, J. A. Hollingsworth, J. Am. Chem. Soc. 2008, 130, 4879.

[3] H. Shen, X. Bai, A. Wang, H. Wang, L. Qian, Y. Yang, A. Titov, J. Hyvonen, Y. Zheng, L. S. Li, Adv. Funct. Mater. 2014, 24, 2367.

[4] J. S. Steckel, J. P. Zimmer, S. Coe-Sullivan, N. E. Stott, V. Bulović, M. G. Bawendi, Angew. Chem. Int. Ed. 2004, 43, 2154.

[5] X. Yang, D. Zhao, K. S. Leck, S. T. Tan, Y. X. Tang, J. Zhao, H. V. Demir, X. W. Sun, Adv. Mater. 2012, 24, 4180.

[6] B. Chen, H. Zhong, W. Zhang, Z. Tan, Y. Li, C. Yu, T. Zhai, Y. Bando, S. Yang, B. Zou, Adv. Funct. Mater. 2012, 22, 2081.

[7] X. Zhang, Y. Zhang, Y. Wang, S. Kalytchuk, S. V. Kershaw, Y. Wang, P. Wang, T. Zhang, Y. Zhao, H. Zhang, T. Cui, Y. Wang, J. Zhao, W. W. Yu, A. L. Rogach, ACS Nano 2013, 7, 11234.

[8] J. Lim, S. Jun, E. Jang, H. Baik, H. Kim, J. Cho, Adv. Mater. 2007, 19, 1927.

[9] K.-H. Lee, J.-H. Lee, W.-S. Song, H. Ko, C. Lee, J.-H. Lee, H. Yang, ACS Nano 2013, 7, 7295

[10] K.-H. Lee, J.-H. Lee, H.-D. Kang, B. Park, Y. Kwon, H. Ko, C. Lee, J. Lee, H. Yang, ACS Nano 2014, 8, 4893.
[11] V. L. Colvin, M. C. Schlamp, A. P. Alivisatos, Nature 1994, 370, 354.

[12] X. Dai, Z. Zhang, Y. Jin, Y. Niu, H. Cao, X. Liang, L. Chen, J. Wang, $X$. Peng, Nature 2014, 515, 96.

[13] Y. Yang, Y. Zheng, W. Cao, A. Titov, J. Hyvonen, J. R. Manders, J. Xue, P. H. Holloway, L. Qian, Nat. Photonics 2015, 9, 259.

[14] B. S. Mashford, M. Stevenson, Z. Popovic, C. Hamilton, Z. Zhou, C. Breen, J. Steckel, V. Bulovic, M. Bawendi, S. Coe-Sullivan, P. T. Kazlas, Nat. Photonics 2013, 7, 407.

[15] Y. Shirasaki, G. J. Supran, M. G. Bawendi, V. Bulović, Nat. Photonics 2013, 7, 13.

[16] H. Shen, W. Cao, N. T. Shewmon, C. Yang, L. S. Li, J. Xue, Nano Lett. 2015, 15, 1211.

[17] W. K. Bae, J. Lim, D. Lee, M. Park, H. Lee, J. Kwak, K. Char, C. Lee, S. Lee, Adv. Mater. 2014, 26, 6387.

[18] H. Choi, S.-J. Ko, Y. Choi, P. Joo, T. Kim, B. R. Lee, J.-W. Jung, H. J. Choi, M. Cha, J.-R. Jeong, I.-W. Hwang, M. H. Song, B.-S. Kim, J. Y. Kim, Nat. Photonics 2013, 7, 732.

[19] Y. Xiao, J. P. Yang, P. P. Cheng, J. J. Zhu, Z. Q. Xu, Y. H. Deng, S. T. Lee, Y. Q. Li, J. X. Tang, Appl. Phys. Lett. 2012, 100, 013308.

[20] A. Kumar, R. Srivastava, P. Tyagi, D. S. Mehta, M. N. Kamalasanan, Org. Electron. 2012, 13, 159.

[21] A. Kumar, R. Srivastava, D. S. Mehta, M. N. Kamalasanan, Org. Electron. 2012, 13, 1750.

[22] X. Gu, T. Qiu, W. Zhang, P. K. Chu, Nanoscale Res. Lett. 2011, G, 199.

[23] B.-H. Kim, C.-H. Cho, J.-S. Mun, M.-K. Kwon, T.-Y. Park, J. S. Kim, C. C. Byeon, J. Lee, S.-J. Park, Adv. Mater. 2008, 20, 3100.

[24] Y. Jin, X. Gao, Nat. Nanotechnol. 2009, 4, 571.

[25] W. Ji, P. Jing, J. Zhao, J. Mater. Chem. C 2013, 1, 470.

[26] E. Cohen-Hoshen, G. W. Bryant, I. Pinkas, J. Sperling, I. Bar-Joseph, Nano Lett. 2012, 12, 4260.

[27] D. Nepal, L. F. Drummy, S. Biswas, K. Park, R. A. Vaia, ACS Nano, 2013, 7, 9064

[28] T. Ozel, S. Nizamoglu, M. A. Sefunc, O. Samarskaya, I. O. Ozel, E. Mutlugun, V. Lesnyak, N. Gaponik, A. Eychmuller, S. V. Gaponenko, H. V. Demir, ACS Nano, 2011, 5, 1328.

[29] O. Kulakovich, N. Strekal, A. Yaroshevich, S. Maskevich, S. Gaponenko, I. Nabiev, U. Woggon, M. Artemyev, Nano Lett. 2002, 2, 1449.

[30] P. Pavaskar, I.-K. Hsu, J. Theiss, W. H. Hung, S. B. Cronin, J. Appl. Phys. 2013, 113, 034302.

[31] B. Peng, Z. Li, E. Mutlugun, P. L. Hernández-Martínez, D. Li, Q. Zhang, Y. Gao, H. V. Demir, Q. Xiong, Nanoscale, 2014, 6, 5592.

[32] X. Yang, K. Dev, J. Wang, E. Mutlugun, C. Dang, Y. Zhao, S. Liu, Y. Tang, S. T. Tan, X. W. Sun, H. V. Demir, Adv. Funct. Mater. 2014, 24, 5977.

[33] J. Kwak, W. K. Bae, D. Lee, I. Park, J. Lim, M. Park, H. Cho, H. Woo, D. Y. Yoon, K. Char, S. Lee, C. Lee, Nano Lett. 2012, 12, 2362.

[34] A. O. Govorov, J. Lee, N. A. Kotov, Phys. Rev. B 2007, 76, 125308.

[35] A. O. Govorov, G. W. Bryant, W. Zhang, T. Skeini, J. Lee, N. A. Kotov, J. M. Slocik, R. R. Naik, Nano Lett. 2006, 6, 984.

[36] D. V. Guzatov, S. V. Vaschenko, V. V. Stankevich, A. Y. Lunevich, Y. F. Glukhov, S. V. Gaponenko, J. Phys. Chem. C 2012, 116, 10723.

[37] Y. Zhao, J. Zhang, S. Liu, Y. Gao, X. Yang, K. S. Leck, A. P. Abiyasa, Y. Divayana, E. Mutlugun, S. T. Tan, Q. Xiong, H. V. Demir, X. W. Sun, Org. Electron. 2014, 5, 871.

[38] B. Wu, X. Wu, C. Guan, K. F. Tai, E. K. L. Yeow, H. J. Fan, N. Mathews, T. C. Sum, Nat. Commun. 2013, 4, 2004.

[39] W. K. Bae, J. Kwak, J. Lim, D. Lee, M. K. Nam, K. Char, C. Lee, S. Lee, Nano Lett. 2010, 10, 2368.

[40] L. Qian, Y. Zheng, J. Xue, P. H. Holloway, Nat. Photonics 2011, 5, 543. 